\title{
A FUNCTIONAL DECOMPOSITION OF FINITE BANDWIDTH REPRODUCING KERNEL HILBERT SPACES
}

\author{
Gregory T. Adams And Nathan A. WAgner
}

Abstract. In this work, we consider "finite bandwidth" reproducing kernel Hilbert spaces which have orthonormal bases consisting of certain polynomials. We provide general conditions based on a matrix recursion that guarantee such spaces contain a functional multiple of the Hardy space. In a particular case, we obtain an explicit functional decomposition of these spaces that greatly generalizes a previous result in the tridiagonal case due to Adams and McGuire. We also prove that multiplication by $z$ is a bounded operator on these spaces and that they contain the polynomials.

Mathematics subject classification (2020): 46E22, 47B32.

Keywords and phrases: Reproducing kernel Hilbert spaces, multiplication operators, Douglas' Range Inclusion Lemma, Hardy space.

\section{REFERENCES}

[1] Gregory T. Adams, Paul J. McGuire, Vern I. Paulsen, Analytic reproducing kernels and multiplication operators, Illinois J. Math. 36 (1992), no. 3, 404-419.

[2] Gregory T. Adams, Paul J. McGuire, Analytic tridiagonal reproducing kernels, J. London Math. Soc. (2) 64 (2001), no. 3, 722-738.

[3] Gregory T. Adams, Paul J. McGuire, A class of tridiagonal reproducing kernels, Oper. Matrices 2 (2008), no. 2, 233-247.

[4] N. AronsZajn, Theory of reproducing kernels, Trans. Amer. Math. Soc. 68 (1950), 337-404.

[5] William Y. C. Chen, James D. Louck, Interpolation for symmetric functions, Adv. Math. 117 (1996), no. 1, 147-156.

[6] R. G. Douglas, On majorization, factorization, and range inclusion of operators on Hilbert space, Proc. Amer. Math. Soc. 17 (1966), 413-415.

[7] VERN I. PAULSEN, An introduction to the theory of reproducing kernel Hilbert spaces, http://www.math.uh.edu/ vern/rkhs.pdf (2009).

[8] AlLEN L. SHIELDS, Weighted shift operators and analytic function theory, Topics in operator theory, pp. 49-128. Math. Surveys, No. 13, Amer. Math. Soc., Providence, R. I., 1974.

[9] Cody Stockdale, Analysis of Five-Diagonal Reproducing Kernels, Honors thesis, Bucknell University, 2015. 